\title{
Cost-effectiveness of a stepped care intervention to prevent depression and anxiety in late life: randomised trial
}

Petronella van't Veer-Tazelaar, Filip Smit, Hein van Hout, Patricia van Oppen, Henriette van der Horst, Aartjan Beekman and Harm van Marwijk

\section{Background}

There is an urgent need for the development of costeffective preventive strategies to reduce the onset of mental disorders.

\section{Aims}

To establish the cost-effectiveness of a stepped care preventive intervention for depression and anxiety disorders in older people at high risk of these conditions, compared with routine primary care.

\section{Method}

An economic evaluation was conducted alongside a pragmatic randomised controlled trial (ISRCTN26474556).

Consenting individuals presenting with subthreshold levels of depressive or anxiety symptoms were randomly assigned to a preventive stepped care programme $(n=86)$ or to routine primary care $(n=84)$.

\section{Results}

The intervention was successful in halving the incidence rate of depression and anxiety at $€ 563$ ( $£ 412$ ) per recipient and $€ 4367$ (£3196) per disorder-free year gained, compared with routine primary care. The latter would represent good value for money if the willingness to pay for a disorder-free year is at least $€ 5000$.

\section{Conclusions}

The prevention programme generated depression- and anxiety-free survival years in the older population at affordable cost.

\section{Declaration of interest}

None.
Preventing depression and anxiety in later life is important from both a public health and an economic point of view. ${ }^{1-3}$ The type of trial described in this paper (comparing stepped care with usual care) differs from conventional stepped care projects (which aim at cost reduction), since the stepped care model is expected to be more expensive even though it is designed to deliver the 'extra' services as efficiently as possible. In an earlier study it was demonstrated that the intervention was successful in reducing the incidence of anxiety and depression by $50 \% .{ }^{4}$ However, the substantial involvement of nursing staff makes the cost-effectiveness of such an intervention debatable.

\section{Method}

\section{Participants and procedures}

The cost-effectiveness analysis was conducted alongside a randomised prevention trial in The Netherlands, comparing clinical outcomes and resource use between two groups of elderly participants with subthreshold depression or anxiety. The trial was registered with the International Standard Randomised Controlled Trials Registry (ISRCTN26474556). The intervention group followed a stepped care programme to prevent the onset of full-blown depressive or anxiety disorders (indicated prevention). ${ }^{5}$ The control group received routine primary care. The trial has been described in detail elsewhere. ${ }^{4}$ In brief, consenting participants were randomised with equal probability to the intervention or to routine primary care in blocks of four by an independent statistician. To be included in the trial, participants had to be at least 75 years old, with a score of 16 or higher on the Center for Epidemiologic Studies Depression (CES-D) scale, but did not meet DSM-IV diagnostic criteria for depressive disorders (major depressive disorder or dysthymic disorder) or anxiety disorders (panic disorder, agoraphobia, social phobia or generalised anxiety disorder), as measured with the Mini International Neuropsychiatric Interview (MINI). A total of 325 people initially agreed to participate, of whom $170(52 \%)$ met the inclusion criteria. Of the remainder, $80(52 \%)$ did not meet the inclusion criteria and $50(32 \%)$ withdrew their consent prior to randomisation (Fig. 1).

\section{Intervention}

The intervention was designed according to the principles of stepped care and outcome management, thus offering the possibility of a flexible step up to more intensive care if necessary. The intervention was structured in cycles of 3 months, and consisted of four steps: watchful waiting, bibliotherapy, problem-solving treatment and antidepressant medication. Monitoring depression symptom severity with the CES-D every 3 months made it possible to evaluate the outcome of each step. Based on the CES-D scores, decisions were made to return to watchful waiting or to step up to more intensive care. A CES-D score of 16 or higher indicated that the next step should be implemented, and this was then discussed with the participant.

\section{Main clinical outcome}

Trained interviewers, masked to the randomisation status of the participants, assessed the MINI/DSM-IV diagnostic status of depressive and anxiety disorders at baseline and at the 6-month and 12-month follow-up assessments. Participants who did not meet the diagnostic criteria for any of the selected disorders at either follow-up point were assumed to have had a disorder-free year, which was the main outcome of the study. 


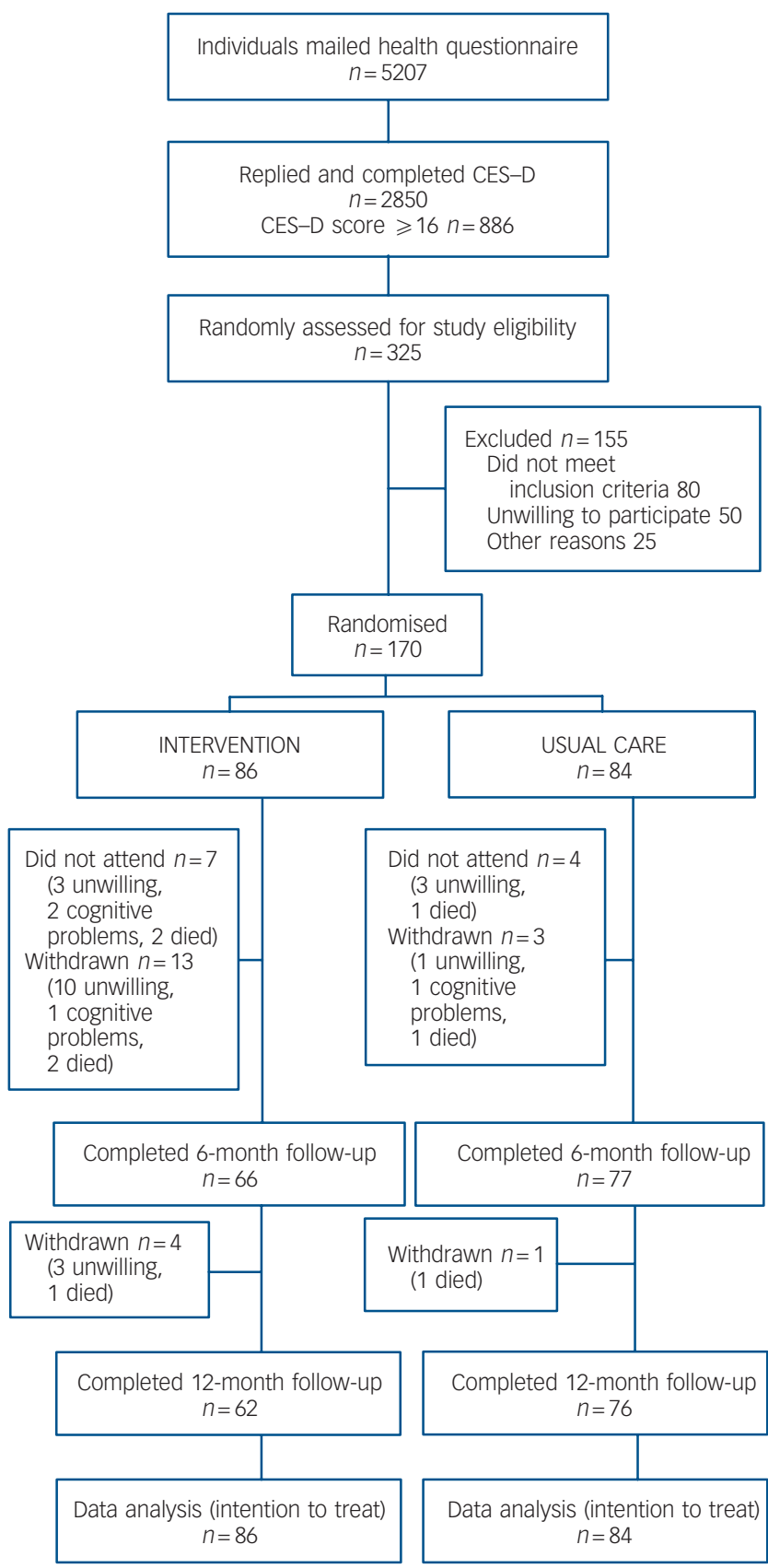

Fig. 1 Flowchart of participants in the trial (CES-D, Center for Epidemiologic Studies - Depression scale).

\section{Resource use and costing}

The study was designed from a societal perspective, and therefore the costs of healthcare uptake and patient out-of-pocket expenses were included. Production losses in paid work were non-existent owing to the age of the study population. Data on resource use related to healthcare uptake were collected with the Trimbos and Institute of Medical Technology Assessment Questionnaire for Costs Associated with Psychiatric Illness (TIC-P), which is the most commonly used healthcare uptake assessment interview in The Netherlands. ${ }^{6}$ To calculate the costs, units of resource use were multiplied by their appropriate full economic cost according to the Dutch guidelines for health economic evaluations. ${ }^{7}$ These costs were originally calculated for the reference year 2003, but were indexed for the year 2007 based on the Statistics Netherlands consumer price index (Table 1). To these we added the costs of medication (antidepressants, anxiolytics and hypnotics), calculated as the price per standard daily dose (obtained from The Netherlands Pharmacotherapeutic Compass; www.fk.cvz.nl), plus $6 \%$ value added tax (not deductible for patients), multiplied by the number of prescription days, plus the pharmacy dispensing costs of $€ 6.45$ per prescription. Finally, direct non-medical costs were computed as the expenses incurred by the participants travelling to receive professional help, and their loss of leisure time, at $€ 8.78$ per hour. ${ }^{7}$ The time that informal caregivers (friends, neighbours, family) might have spent running errands for the participants was also valued at $€ 8.78$ per hour.

The costs in euros $(€)$ can be converted to pounds sterling $(\mathfrak{E})$ using the purchasing power parities reported by the Organisation for Economic Cooperation and Development, which convert currencies taking into account the differential buying power across countries. For the reference year 2007, $€ 1$ in The Netherlands was equal to a little over $\mathfrak{E} 0.73$.

\section{Per patient intervention costs}

The per patient intervention costs were calculated for each of the treatment steps separately. The average cost of the first screening step was estimated to be $€ 26$ ( $£ 19$ ). This step consisted of watchful waiting, and involved sending the CES-D questionnaire by post $(€ 1)$, a telephone call made by a specialised nurse $(€ 16)$, 10 min of administration time $(€ 7)$ and the time needed by the participant to complete the questionnaire $(€ 2)$.

The second step consisted of bibliotherapy, with the active involvement of district (enrolled) nurses. The average costs of this step were estimated to be $€ 259.25$ ( $£ 189.77$ ). The costs of this step were calculated as the costs of the time needed by the nurses for an average of four $15 \mathrm{~min}$ sessions (€42.73), corresponding travel expenses of $4 \times 30 \mathrm{~min}$ (€97.84), plus administration time $4 \times 10 \mathrm{~min}$ ( $€ 28.48$ ). Added to these costs was that of screening, which was similar to the screening described in the first step (€26). The participants incurred costs for a folder, a self-help book and worksheets (€29.00). Their time costs were valued as leisure time for four $15 \mathrm{~min}$ sessions (totalling €8.80), plus an average of $3 \mathrm{~h}$ spent reading the bibliotherapeutic material ( $€ 26.40$ ).

The third step involved problem-solving treatment provided by (registered) district community psychiatric nurses. The costs of this step were calculated to total $€ 638.24$ ( $£ 467.19$ ). These costs consisted of the time needed by the nurses for an average of four $45 \mathrm{~min}$ sessions (€285.52), plus administration time $(4 \times 15 \mathrm{~min}$, $€ 95.16)$, completion of worksheets (€2) and travelling time $(4 \times 30 \mathrm{~min}, € 203.16)$. To these were added the costs of the time needed by the participant for four $45 \mathrm{~min}$ sessions (€26.40) and the cost of the screening (€26).

The last step involved screening (€26) and referral to a general practitioner if necessary. The total cost of this step was calculated to be $€ 59.36$ ( $£ 43.45$ ). This was calculated as the cost of writing a letter of referral to the general practitioner, plus the direct medical costs of a $10 \mathrm{~min}$ visit to the general practitioner (€21.36), plus the participant's out-of-pocket expenses for travel and the time needed to make this visit $(€ 10)$.

\section{Statistical analysis}

All analyses were carried out on an intention-to-treat basis. Therefore, all participants were analysed in the group to which they were randomised, and missing data were imputed, using two different techniques for missing clinical end-points and missing cost data. Missing clinical end-points (MINI/DSM diagnostic status) were replaced by their most likely value, taking 
Table 1 Direct medical and non-medical costs according to type of healthcare

\begin{tabular}{|c|c|c|c|c|}
\hline \multirow[b]{2}{*}{ Health service type } & \multicolumn{2}{|c|}{ Direct medical costs } & \multicolumn{2}{|c|}{ Direct non-medical costs } \\
\hline & Unit & Unit cost, $^{\mathrm{a}} €$ & Zones, hours ${ }^{\mathrm{b}}$ & Unit cost, ${ }^{\mathrm{c}} €$ \\
\hline General practitioner $^{\mathrm{d}}$ & Contact & 21.36 & 1 zone, $1 \mathrm{~h}$ & 10.00 \\
\hline Medical specialist, out-patient department & Consultation & 59.23 & 4 zones, $3 \mathrm{~h}$ & 36.40 \\
\hline Social worker & Contact & 47.59 & 2 zones, $3 \mathrm{~h}$ & 29.40 \\
\hline Physiotherapist & Contact & 24.06 & 2 zones, $2 \mathrm{~h}$ & 20.60 \\
\hline Community psychiatric nurse & Contact & 47.59 & 4 zones, $3 \mathrm{~h}$ & 36.40 \\
\hline Private practice psychotherapist & Session & 80.38 & 2 zones, $2 \mathrm{~h}$ & 20.60 \\
\hline Regional mental health service & Contact & 131.14 & 4 zones, $3 \mathrm{~h}$ & 36.40 \\
\hline Alternative treatment & Contact & 40.79 & 2 zones, $1 \mathrm{~h}$ & 11.80 \\
\hline Meals on wheels & Meal & 4.00 & NA & 0.00 \\
\hline Social activities ${ }^{e}$ & Day & 11.88 & NA & 0.00 \\
\hline Home care & Hour & 22.95 & NA & 0.00 \\
\hline Informal care (family, friends) ${ }^{f}$ & Hour & 8.78 & NA & 0.00 \\
\hline \multicolumn{5}{|c|}{$\begin{array}{l}\text { NA, not applicable. } \\
\text { a. Integral unit cost prices (indexed for 2007). } \\
\text { b. Based on average distances (in special tariff taxi zones) and travel + waiting + treatment times (in h) for receiving treatment (indexed for 2007). } \\
\text { C. Cost of return journey across } 2 \text { taxi zones, reduced tariff } € 3.00 ; 4 \text { taxi zones } € 10.00 ; \text {; hours costed at } € 8.80 \text { per hour (indexed for } 2007 \text { ) }{ }^{7} \\
\text { d. Average direct medical cost price per surgery contact is } € 21.36 \text {. However, the majority of contacts of older people with their general practitioner concern repeat prescriptions } \\
\text { (cost } € 10.10 \text { ), and although the type of contact in this study is unknown, we may assume that the real direct cost is probably lower. } \\
\text { e. Minibus transport is included in the day care and social activities price. } \\
\text { f. Valued as domestic help (indexed for 2007). }\end{array}$} \\
\hline
\end{tabular}

into account the mechanism that generated the missing values. Predictors of outcome and 'missingness' were identified by logistic regression analysis. Predictors of outcome help us to obtain the most exact values of the outcome variable, and predictors of 'missingness' help us to correct for the bias that may be caused by differential loss to follow-up. The statistically significant predictors were used in a regression imputation (as implemented in Stata version 8.2) to obtain the required predicted values. ${ }^{4}$

Missing cost data were imputed according to the last observation carried forward method, because this provided the most conservative estimates for our data, thus strengthening the null hypothesis of equivalence of both the cost and the effectiveness of the intervention compared with routine primary care. This method was considered to be the most conservative approach for the calculation of costs in this study, since there was a visible trend from higher costs at baseline towards lower costs at the subsequent measurement points.

The imputed data were then analysed. First, it was determined how many participants had MINI/DSM-IV depression or anxiety in each of the groups at follow-up, to assess the risk of developing depression or anxiety. The probability of not developing depression or anxiety is equal to 1 risk, and this was interpreted as the likelihood of a depression/anxiety-free year. The incremental effectiveness was computed as the difference in the probability of a disorder-free period between the intervention group and the control group.

The mean total costs for each of the groups were calculated, both at baseline and at follow-up. The cumulative annual differences in costs were computed to obtain the increase (or decrease) in costs over time in each of the groups. The incremental costs could then be calculated as the difference in the costs of the intervention group minus the costs of the routine primary care group. Both the incremental costs and the incremental effects were used to calculate the incremental cost-effectiveness ratio (ICER), the incremental cost of gaining one disorder-free year. This was carried out according to the relevant guidelines for health economic evaluations. ${ }^{8-10}$

Non-parametric bootstraps were used to simulate 5000 ICERs, which were plotted on the cost-effectiveness plane (see Fig. 2). In this way the degree of uncertainty associated with the ICER is visualised. ${ }^{11}$ To be more precise, each simulated ICER can be plotted in one of the four quadrants of the ICER plane. In the north-east quadrant the intervention produces superior health gains at additional costs, compared with routine primary care. In the north-west quadrant inferior health gains are produced at additional costs; clearly, for the intervention this is the worst possible outcome, and the intervention is then said to be 'dominated' by routine primary care. In the south-west quadrant inferior health gains are produced, but there are some cost savings. Finally, in the south-east quadrant the intervention produces superior health gains (compared with the other group) and does so for less costs; the intervention is then said to 'dominate' routine primary care. From a decision analysis point of view the ICER plane helps to ascertain the probability that the intervention falls within one particular quadrant, and these probabilities are reported.

It is often seen that a new intervention has a high probability of falling within the north-east quadrant because the intervention is successful in generating better health outcomes, albeit at higher cost. However, to decide whether the intervention offers good value for money, another piece of information is required: the willingness to pay (WTP) for an additional unit of health gained. The WTP ceiling, indicating a cap on the costs that one is willing to pay for an additional disorder-free year, however, is an unknown quantity. We therefore used a series of WTP ceilings, and calculated the probability that the intervention is more acceptable than routine primary care from a cost-effectiveness point of view for each of these ceilings. ${ }^{12}$ This can be visualised in an ICER acceptability curve, with increasing WTP levels on the horizontal axis, and on the vertical axis the probability that the intervention is more acceptable from a cost-effectiveness point of view, given the corresponding WTP ceiling (see Fig. 3).

\section{Sensitivity analysis}

The single most important cost driver was the intervention, and in particular the cost of the nurses' time. It is also a cost driver that is surrounded by some uncertainty, specifically with regard to steps 
2 and 3 of the programme: for instance, the optimal and most cost-effective number of sessions for bibliotherapy and problemsolving treatment in later life is not yet known. To ascertain the robustness of our findings, all the analyses were repeated for six different cost scenarios: in one scenario the nursing cost was decreased by $20 \%$, and in the other five this cost was repeatedly increased by $20 \%$.

\section{Results}

Most of the participants were women (74\%), and had a mean age of 81.4 years (s.d.=3.7) (Table 2). Approximately $30 \%$ of the participants were married or living with a partner, and $73 \%$ had a level of education that was lower than or equivalent to secondary education. Half the participants had more than two chronic illnesses (such as ischaemic heart disease and arthritis). At baseline the mean CES-D score was 21.6 (s.d. = 5.1).

\section{Incremental costs}

The mean total costs at baseline were $€ 601$ (s.d. $=559$ ) in the intervention group and $€ 618$ (s.d. $=531$ ) in the usual care group, indicating that randomisation produced evenly distributed costs across the conditions at baseline. The mean total costs were direct medical costs, the costs of treatment offered by a broad range of both formal and informal caregivers, plus direct non-medical costs incurred by the participants when they travelled to the caregivers. Table 3 presents the per capita costs accumulated over 1 year in each of the groups.

The intervention costs per participant were $€ 26.00$ for step 1, $€ 259.25$ for step 2, €638.24 for step 3 and $€ 59.36$ for step 4 . However, the aim of the stepped care programme was to provide more intensive and more expensive interventions only if the less intensive interventions were ineffective. All $86(100 \%)$ participants attended step 1; 79 (92\%) attended step 2; 38 (44\%) attended step 3; and 25 (29\%) participants attended step 4. On average the intervention costs amounted to $€ 563.42$ ( $£ 412.42$ ). The total incremental costs are calculated as the difference between the two groups, (€2985-€2453) =€532, indicating that the intervention was more costly than routine primary care.

\section{Incremental effectiveness}

The intervention halved the 12-month incidence of depressive and anxiety disorders (relative risk $0.49,95 \%$ CI $0.24-0.98$ ). In the intervention group, 76 of the 86 participants did not develop a MINI/DSM-IV major depression or anxiety disorder during the 12-month period. The probability of a depression/anxiety-free year was therefore $76 / 86=0.88$. In the routine primary care group the probability of not developing depression or anxiety was

\begin{tabular}{|c|c|c|c|}
\hline & $\begin{array}{l}\text { Experimental } \\
\text { group } \\
(n=86)\end{array}$ & $\begin{array}{l}\text { Usual care } \\
\text { group } \\
(n=84)\end{array}$ & $\begin{array}{c}\text { Total } \\
(n=170)\end{array}$ \\
\hline Female gender, $n(\%)$ & $60(70)$ & $65(77)$ & $125(74)$ \\
\hline Age, years: mean (s.d.) & $81.8(3.8)$ & $81.1(3.5)$ & $81.4(3.7)$ \\
\hline Married or living with partner, $n(\%)$ & $26(30)$ & $24(29)$ & $50(29)$ \\
\hline Education beyond high school, $n$ (\%) & $24(28)$ & $22(26)$ & $46(27)$ \\
\hline $\begin{array}{l}\text { Rural residence } \\
(<10000 \text { inhabitants), } n \text { (\%) }\end{array}$ & $36(42)$ & $39(46)$ & $75(44)$ \\
\hline Chronic diseases (>2), $n$ (\%) & $36(42)$ & $45(54)$ & $81(48)$ \\
\hline CES-D score, mean (s.d.) & $21.2(5.0)$ & $22.1(5.2)$ & $21.6(5.1)$ \\
\hline
\end{tabular}

$64 / 84=0.76$. The incremental effectiveness was calculated as the difference between the probabilities of a beneficial outcome in each of the groups, i.e. $0.88-0.76=0.12$ (95\% CI 0.01-0.24), and was statistically significant at $P=0.037$. The incremental effectiveness was the clinical parameter of interest in the remainder of this study. Its inverse - the number needed to treat - was $1 / 0.12=8.3$, indicating that an extra disorder-free year was gained in one out of every eight participants in the experimental group.

\section{Incremental cost-effectiveness}

The incremental cost-effectiveness ratio was calculated as $\mathrm{ICER}=\left(\right.$ Cost $_{\exp }-$ Costs $\left._{\mathrm{ctr}}\right) /\left(\right.$ Effects $_{\exp }-$ Effects $\left._{\mathrm{ctr}}\right)$, where Costs is the average annual per capita cost and Effects is the percentage of participants who did not develop depression or anxiety in the two groups (subscripted exp and ctr respectively). In this study, in which the incremental costs were $€ 532$ and the incremental effects were 0.12 , the mean ICER was estimated at $€ 532 /$ $0.12=€ 4367$ for a depression/ anxiety-free year. Using the 5000 bootstrap estimates, the median ICER could also be estimated as $€ 4297$ ( $95 \%$ CI -899 to 27077 ).

In the incremental cost-effectiveness plane (Fig. 2) each dot indicates one simulated ICER. Of these, $96 \%$ fell in the north-east quadrant, indicating a probability of $96 \%$ that by applying the intervention a health gain is produced, but at additional costs. In addition, $1 \%$ of the ICERs fell in the north-west quadrant (dominated) and another $3 \%$ in the south-east quadrant (dominates).

\section{Acceptability}

The incremental cost-effectiveness acceptability curve suggests that when the willingness to pay for a depression/anxiety-free year is $€ 5000$, $€ 10000$ or $€ 20000$, then this preventive programme would have a probability of being regarded as more cost-effective than routine primary care by $57 \%, 86 \%$ and $94 \%$ respectively (Fig. 3).

\section{Sensitivity analysis}

The intervention costs mainly consist of the costs of the nurses and their travelling expenses for visits to their clients. Jointly, these

Table 3 Annual per capita costs according to study group

\begin{tabular}{|c|c|c|c|}
\hline & \multicolumn{3}{|c|}{ Costs, $€^{\mathrm{a}}$} \\
\hline & $\begin{array}{l}\text { Experimental } \\
\text { group (E) } \\
(n=86) \\
\text { Mean (s.d.) }\end{array}$ & $\begin{array}{l}\text { Usual care } \\
\text { group (C) } \\
(n=84) \\
\text { Mean (s.d.) }\end{array}$ & $\begin{array}{c}\text { Difference } \\
\text { E - C }(95 \% \mathrm{Cl})\end{array}$ \\
\hline Intervention & $563(361)$ & & 563 (486 to 641 ) \\
\hline General practitioner & $131(118)$ & $101(86)$ & 29 (-2 to 60$)$ \\
\hline Medication $^{\mathrm{b}}$ & $25(58)$ & $20(48)$ & $5(-12$ to 21$)$ \\
\hline Psychological support ${ }^{C}$ & 89 (294) & $76(229)$ & $13(-67$ to 93$)$ \\
\hline Home care & 1367 (1263) & 1497 (1415) & $-130(-536$ to 276$)$ \\
\hline Meals on wheels & 63 (198) & 66 (192) & $-3(-62$ to 56$)$ \\
\hline Social activities & $2(14)$ & 31 (158) & $-29(-63$ to 6$)$ \\
\hline Other medical $^{d}$ & 257 (314) & 245 (303) & 12 (-82 to 105$)$ \\
\hline Informal care & 208 (394) & $171(280)$ & 37 (-67 to 140$)$ \\
\hline Direct medical costs & $2141(1566)$ & 2208 (1689) & $-67(-551$ to 418$)$ \\
\hline Direct non-medical costs & $280(303)$ & $245(251)$ & 35 (-49 to 119$)$ \\
\hline Total cost & 2985 (1711) & 2453 (1828) & $532(-4$ to 1068$)$ \\
\hline \multicolumn{4}{|c|}{$\begin{array}{l}\text { a. Costs at } 2007 \text { prices. } \\
\text { b. Antidepressants, anxiolytics and hypnotics. } \\
\text { c. Contacts with community psychiatric nurse, psychologist or social worker, } \\
\text { not part of the intervention. } \\
\text { d. Medical specialist out-patient consultation, physiotherapist, alternative treatments. }\end{array}$} \\
\hline
\end{tabular}




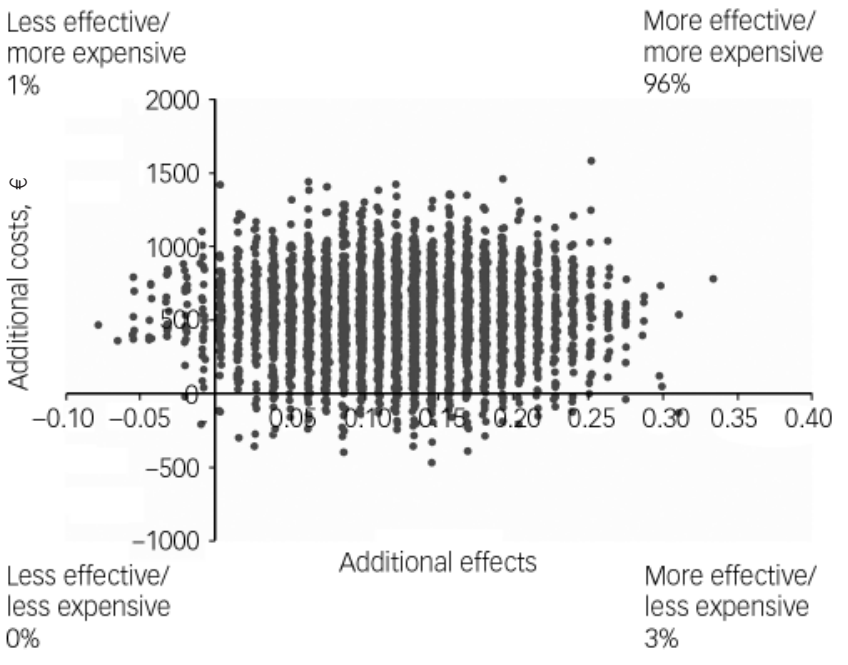

Fig. 2 Distribution of 5000 bootstrapped incremental cost-effectiveness ratios in the cost-effectiveness plane: primary analysis.

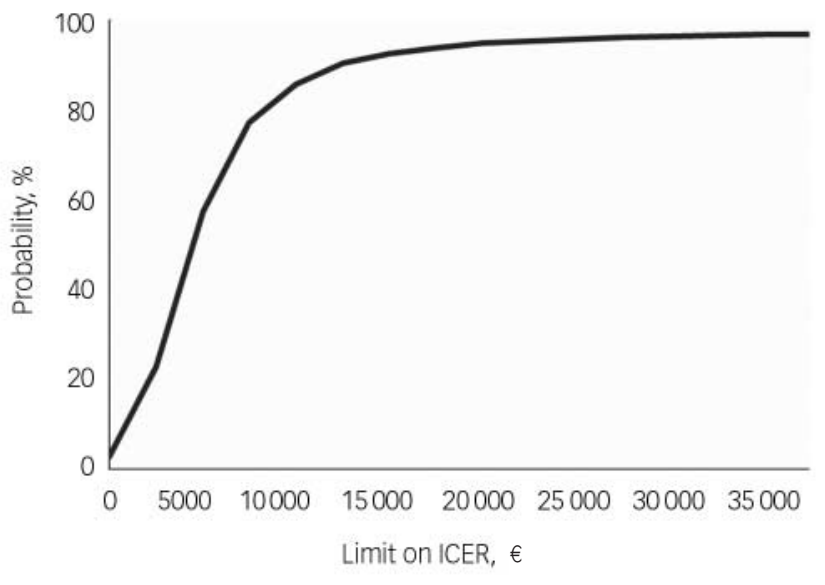

Fig. 3 Cost-effectiveness acceptability curve: probability that the intervention is acceptable ( $y$-axis) relative to routine primary care, given varying thresholds for willingness to pay ( $x$-axis), based on 5000 bootstrap replications (ICER, incremental cost-effectiveness ratio). expenses therefore constitute the most important cost driver. However, there is uncertainty surrounding these values. We therefore conducted sensitivity analyses based first on the estimated nurses' costs minus 20\%, and then plus $20 \%, 40 \%, 60 \%, 80 \%$ and even $100 \%$ to verify the robustness of the results. In the first scenario the median ICER was $€ 4297$ per disorder-free year gained. The median ICER increased to $€ 7499$ if the nurses' costs increased by $100 \%$, an ICER which has a $90 \%$ probability of falling below the willingness to pay ceiling of $€ 20000$. The outcomes of the sensitivity analyses are presented in Table 4. The decrease and increases in ICERs do not affect the overall conclusion in any fundamental way. This indicates that the intervention still produces better health at additional costs, compared with routine primary care.

\section{Discussion}

The intervention was successful in reducing the risk of disorder onset by $50 \%$ compared with routine primary care, at an incremental cost of $€ 532$. A depression/anxiety-free year was achieved on average for $€ 4367$ (median $€ 4297$ ). The probability that the intervention is considered to be more acceptable than routine primary care depends on the willingness to pay for a disorder-free year: at $€ 5000, € 10000$ and $€ 20000$ this likelihood is $57 \%, 85 \%$ and $95 \%$ respectively - suggesting that at WTP ceilings of $€ 5000$ and above the intervention must be regarded as good value for money - but this conclusion is somewhat sensitive to the valuation of the nurses' time.

The incremental costs of the stepped care programme compared with routine primary care in our study appear to be higher than those reported by Willemse et al and Smit et al. ${ }^{13,14}$ Bibliotherapy could generate a depression-free year while producing cost savings compared with routine primary care at $€ 289$ (£212). The incremental costs of the stepped care programme compare favourably with those reported by Simon et al, who compared the incremental cost-effectiveness of stepped collaborative care with usual care for patients with persistent depressive symptoms. ${ }^{15}$ The collaborative care included systematic patient education, an initial visit to a consulting psychiatrist,

\begin{tabular}{|c|c|c|c|c|c|c|c|}
\hline & \multirow{2}{*}{$\begin{array}{c}\text { Standard } \\
\text { stepped care }\end{array}$} & \multicolumn{6}{|c|}{ Adjusted cost of nursing care } \\
\hline & & $-20 \%$ & $+20 \%$ & $+40 \%$ & $+60 \%$ & $+80 \%$ & $+100 \%$ \\
\hline Cost, $€^{a}$ & 532 & 449 & 615 & 697 & 780 & 863 & 945 \\
\hline Effect & 0.12 & 0.12 & 0.12 & 0.12 & 0.12 & 0.12 & 0.12 \\
\hline ICER, €: median $^{\mathrm{b}}$ & 4297 & 3491 & 4819 & 5610 & 6215 & 6896 & 7499 \\
\hline \multicolumn{8}{|l|}{ Distribution on the cost-effectiveness plane } \\
\hline First quadrant (north-east) & 0.96 & 0.93 & 0.97 & 0.98 & 0.98 & 0.98 & 0.98 \\
\hline Second quadrant (inferior: north-west) & 0.01 & 0.02 & 0.02 & 0.01 & 0.02 & 0.02 & 0.02 \\
\hline Third quadrant (south-west) & 0.00 & 0.00 & 0.00 & 0.00 & 0.00 & 0.00 & 0.00 \\
\hline Fourth quadrant (dominant: south-west) & 0.03 & 0.05 & 0.01 & 0.01 & 0.00 & 0.00 & 0.00 \\
\hline \multicolumn{8}{|l|}{ WTP ceiling, \% } \\
\hline$€ 5000$ & 57 & 66 & 51 & 41 & 33 & 28 & 21 \\
\hline$€ 10000$ & 86 & 88 & 82 & 79 & 75 & 71 & 67 \\
\hline$€ 20000$ & 95 & 95 & 94 & 93 & 92 & 90 & 90 \\
\hline
\end{tabular}


2-4 months of shared care provided by a psychiatrist and a primary care physician, and the monitoring of follow-up visits and adherence to medication regimen. The incremental cost-effectiveness was US\$7826 per depression-free year (€6908

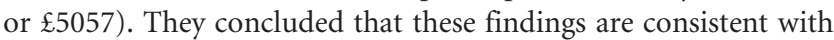
those of other randomised trials. Improving the outcomes of treatment for depression in primary care requires the investment of additional resources, but the return on this investment is comparable to that of many other widely accepted medical interventions. $^{15}$

\section{Cost-effectiveness of the stepped care programme}

Although the stepped care programme appears to be cost-effective, the two active interventions of the programme (steps 2 and 3) may be relatively costly when compared with one-step self-help interventions. Nevertheless, using a stepped care programme can be more cost-effective overall when less costly interventions are offered first. A smaller percentage of the total group of participants would then move on to the more intensive and costly interventions. Precisely this phenomenon was observed in these data and contributed to keeping the cumulative per-patient costs of the stepped care intervention as low as $€ 532$. However, the steps themselves and perhaps a different order of steps deserve closer scrutiny. It is consistently reported that subclinical manifestations are amenable particularly to preventive cognitive-behavioural therapy and problem-solving treatment, as evidenced by a metaanalysis of randomised prevention trials. ${ }^{16}$ Both treatment types help patients to acknowledge their symptoms and encourage them to switch to more active self-management strategies. This makes both treatments promising candidates for prevention and was the reason we chose them. Furthermore, many (older) participants seem to prefer psychotherapy to medication, ${ }^{17}$ which was also a reason for the structure of the stepped care programme in this study. However, since the major cost of the stepped care programme was the nursing time spent in delivering problemsolving treatment in step 3, a cost-saving solution might be to exchange steps 3 and 4 , in this way first accommodating the relative cheapness of a general practitioner prescribing off-patent antidepressants, followed in the next and last step by the dedicated but costly nurse delivering a psychological intervention. It might be worthwhile to study the effects, the costs and the consequences both for the participants and for their general practitioners of such an exchange of steps. We do not know, for instance, to what extent participants in a prevention study would accept preventive drug treatment with antidepressants. In The Netherlands antidepressant medication is often prescribed, but not for preventive purposes, and adherence rates are typically low. Two other (possible) cost-saving approaches might be that participants could choose their own preferred order of steps, or to drop the expensive problem-solving treatment step altogether. However, apart from the costs of the intervention, the effects have an equally important impact on the cost-effect ratio. The effects are known to be dependent on the degree of the patients' acceptance of and adherence to the intervention. Furthermore, the stepped care programme in this study was analysed as a whole, ${ }^{4}$ and the effectiveness of the separate parts of the programme are not known.

\section{Strengths and limitations}

A limitation of this study concerns the fact that attrition might have compromised the representativeness of the sample (24 people withdrew from the intervention group $v .8$ from the routine primary care group). Unwillingness was the reason for 16 of the
24 withdrawals. Three elderly people in our sample withdrew after they had initially given their consent. A further ten people left the study between 1 month and 6 months, and after 6 months another three had done so. The data may point towards self-selection of participation in treatment, or the self-help and problem-solving treatment interventions may have been perceived by the participants as too strenuous relative to the symptom levels, resulting in a lack of perceived need for these interventions. Another limitation may be that reducing or (repeatedly) raising the nursing care cost by $20 \%$ was a somewhat arbitrary choice. It does, however, demonstrate that the results may generalise to other healthcare settings in which salary costs might differ.

The strengths of this study were its randomised design, the real-life integrated preventive care we offered and the 12 months of follow-up. Elsewhere we had already provided evidence that the prevention of late-life depression and anxiety is effective, and, to our knowledge, this is one of the first studies to provide that kind of evidence. Moreover, we were also able to generate some evidence that these favourable results were achieved in a cost-effective way.

\section{Petronella van't Veer-Tazelaar, MA, Department of General Practice, and EMGO Institute for Health and Care Research (EMGO+), Vrije Universiteit (VU) Medical Centre, Amsterdam; Filip Smit, PhD, Trimbos Institute (Netherlands Institute of Centre, Amsterdam; Filip Smit, PhD, Trimbos Institute (Netherlands Institute
Mental Health and Addiction), Utrecht, and Department of Epidemiology and Biostatistics and EMGO+, VU Medical Centre, Amsterdam; Hein van Hout, PhD, Department of General Practice and EMGO+; Patricia van oppen, PhD, Department of Psychiatry and EMGO+; Henriette van der Horst, MD, PhD, Department of General Practice and EMGO+; Aartjan Beekman $\mathrm{MD}, \mathrm{PhD}$, Department of Psychiatry and EMGO+; Harm van Marwijk, MD, PhD, Department of Genera Practice and $\mathrm{EMGO}+$, VU Medical Centre, Amsterdam, The Netherlands.}

Correspondence: Petronella J. van't Veer-Tazelaar, Department of Genera Practice, Institute for Research in Extramural Medicine, VU Medical Centre, Van der Boechorststraat 7, 1081 BT Amsterdam, The Netherlands. Email: pj.vantveer@vumc.n

First received 29 Jun 2009, final revision 6 Nov 2009, accepted 21 Dec 2009

\section{Funding}

This work was financially supported by grant 2620.0003 from The Netherlands Health Research Council.

\section{References}

1 De Beurs E, Beekman AT, van Balkom AJ, Deeg DJ, van Dyck R, van Tilburg W. Consequences of anxiety in older persons: its effect on disability, well-being and use of health services. Psychol Med 1999; 29: 583-93.

2 Beekman AT, Penninx BW, Deeg DJ, de Beurs E, Geerling SW, van Tilburg W. The impact of depression on the well-being, disability and use of services in older adults: a longitudinal perspective. Acta Psychiatr Scand 2002; 105 20-7.

3 Smit F, Cuijpers P, Oostenbrink J, Batelaan N, de Graaf R, Beekman A. Costs of nine common mental disorders: implications for curative and preventive psychiatry. J Ment Health Policy Econ 2006; 9: 193-200.

4 Veer-Tazelaar PJ, van Marwijk HW, van Oppen P, van Hout HP, van der Horst $\mathrm{HE}$, Cuijpers $\mathrm{P}$, et al. Stepped-care prevention of anxiety and depression in late life: a randomized controlled trial. Arch Gen Psychiatry 2009; 66: 297-304.

5 Mrazek PJ, Haggerty RJ (eds). Reducing Risks for Mental Disorders. National Academy Press, 1994

6 Hakkaart-van Roijen L, Van Straten A, Donker M, Tiemens B. Trimbos/iMTA Questionnaire for Costs Associated with Psychiatric IIIness (TiC-P). Erasmus University, 2002.

7 Oostenbrink JB, Bouwmans CAM, Koopmanschap MA, Rutten FFH. Guideline for Economic Evaluations in Healthcare. Institute for Medical Technology Assessment, 2004

8 Langley PC. The November 1995 revised Australian guidelines for the economic evaluation of pharmaceuticals. Pharmacoeconomics 1996; 9 341-52. 
9 Siegel JE, Torrance GW, Russell LB, Luce BR, Weinstein MC, Gold MR. Guidelines for pharmacoeconomic studies. Recommendations from the Panel on Cost Effectiveness in Health and Medicine. Pharmacoeconomics 1997; 11: $159-68$.

10 Torrance GW, Blaker D, Detsky A, Kennedy W, Schubert F, Menon D, et al. Canadian guidelines for economic evaluation of pharmaceuticals. Canadian Collaborative Workshop for Pharmacoeconomics. Pharmacoeconomics 1996; 9: 535-59.

11 Van Hout BA, Al MJ, Gordon GS, Rutten FF. Costs, effects and C/E-ratios alongside a clinical trial. Health Econ 1994; 3: 309-19.

12 Barrett B, Byford S. Acceptable mental health care? A new decision-making tool for cost-effectiveness analysis. Ment Health Res Rev 2003; 9: 39-41.

13 Willemse GR, Smit F, Cuijpers P, Tiemens BG. Minimal-contact psychotherapy for sub-threshold depression in primary care: randomised trial. Br J Psychiatry 2004; 185: 416-21.
14 Smit F, Willemse G, Koopmanschap M, Onrust S, Cuijpers P, Beekman A. cost-effectiveness of preventing depression in primary care patients: randomised trial. Br J Psychiatry 2006; 188: 330-6.

15 Simon GE, Katon WJ, Von Korff M, Unutzer J, Lin EH, Walker EA, et al. Cost-effectiveness of a collaborative care program for primary care patients with persistent depression. Am J Psychiatry 2001; 158: 1638-44.

16 Cuijpers $\mathrm{P}$, Van Straten A, Smit F, Mihalopoulos C, Beekman A. Preventing the onset of depressive disorders: a meta-analytic review of psychological interventions. Am J Psychiatry 2008; 165: 1272-80.

17 Van Schaik DJ, Klijn AF, van Hout HP, van Marwijk HW, Beekman AT, de Haan $\mathrm{M}$, et al. Patients' preferences in the treatment of depressive disorder in primary care. Gen Hosp Psychiatry 2004; 26: 184-9.
Psychiatry in music

\section{Robert Schumann's contribution to the genetics of psychosis}

\section{Katharina Domschke}

Robert Schumann (1810-1856), one of the most influential romantic composers, had a lifelong mental disorder, first manifesting in 1833 as a severe melancholic depressive episode, which recurred several times alternating with phases of 'exaltation' and increasingly also delusional ideas of being poisoned or threatened with metallic items. After a suicide attempt, Schumann was admitted to a mental asylum in Endenich near Bonn and diagnosed with 'psychotic melancholia'. He died in Endenich without having recovered from his mental illness.

Hypothetical diagnoses of Schumann's ailments vary from progressive paralysis to hypertensive encephalopathy, with the most compelling evidence being for either schizophrenia ('dementia praecox'; 'periodic catatonia') or bipolar disorder and bipolar II disorder. Delusional ideas, ideas of reference as well as acoustic hallucinations support a diagnosis on the schizophrenic spectrum. However, the notion of Schumann having a bipolar disorder possibly with psychotic features is substantiated by the undulating course of his illness with distinct depressive and hypomanic phases as well as his recovery from single episodes with full restoration of his musical and compositional abilities.

Given the still ongoing clinical debate about Schumann's diagnosis, a more detailed analysis of his pedigree might help to elucidate the nature of his disease. Therefore, a hypothetical pedigree based on descriptions of Schumann's ancestry (Bienenfeld, E., Archiv für Rassen- und Gesellschaftsbiologie (1932) \& Lindner, A., Genealogie (1974)) is proposed here (Fig. 1)

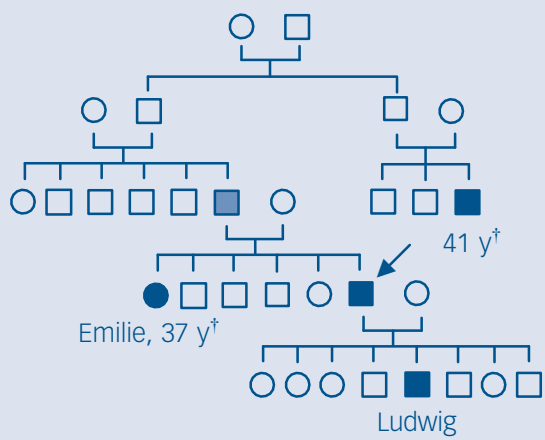

Fig. 1 Robert Schumann's hypothetical pedigree with respect to the segregation of mental disorders

Square - male; circle - female; arrow - index person Robert Schumann; black filling - affected by mental disorder; black/white shadowed - possibly affected by mental disorder; "death by suicide.

Apart from Schumann himself, his sister Emilie experienced 'a never-healing mental disorder, which intermittently carried traits of silent insanity as horrible sequelae of this disease', which retrospectively can most probably be diagnosed as schizophrenia, and died by suicide at the age of 37 . Also, his son Ludwig is reported to have had a severe, probably schizophreniform mental disorder, which necessitated admission to a mental asylum at the age of 28 , where he lived for another 25 years without recovering from his illness. Schumann's father Friedrich August, otherwise successful as a publisher, is described as melancholic and 'sensitive close to insanity'. Finally, a cousin of Schumann's father's died by suicide at the age of 41 , probably having had an affective mental disorder.

Obviously, mental disorders have segregated in Robert Schumann's family, which strengthens the notion of either bipolar disorder or schizophrenia, rather than progressive paralysis, to underlie Schumann's symptomatology. Given that his symptoms cannot conclusively be attributed to either schizophrenia or bipolar disorder and that in his family both psychotic and affective disorders or symptoms can be discerned, a schizophrenia-bipolar spectrum rather than a clear-cut Kraepelinian dichotomy might be proposed for mental disorders segregating in Schumann's family. This is in line with clinical and epidemiological observations of a continuum between schizophrenia and bipolar disorder, both considered complex genetic disorders with similarly high heritability of 85-93\%. Consistently, linkage studies report risk loci common for schizophrenia and bipolar disorder, particularly on chromosomes 8p22. $10 p 14,13 q 32,18 p 11,22 q 11$ and $6 p$ and there is molecular genetic evidence of candidate genes influencing the disease risk of both schizophrenia and bipolar disorder: G72(DAOA)/G30 on chromosome 13q48, DISC1 at 1q42, neuregulin 1 (NRG1) at 8p21-22 and catechol-0-methyltransferase (COMT) on chromosome 22q11. Additionally, a linkage study analysing families of probands meeting criteria for schizoaffective disorder strongly supports the existence of loci that provide specific susceptibility to psychosis with both schizophrenic and bipolar features at chromosome 1q42 and possibly also at 22q11 and 19p13. Thus, given an aggregation of both affective and psychotic disorders in Robert Schumann's family and his own symptoms with both affective and psychotic features, here schizoaffective disorder or a 'schizoaffective spectrum phenotype' with a major genetic susceptibility conferred by overlapping sets of genes is proposed as a differential diagnosis of Schumann's mental disorder. 